\title{
Heterosis Studies for Grain Yield and its Contributing Traits in Pearl millet (Pennisetum glaucum (L.) R. Br.) under Different Sowing Conditions
}

\author{
Anita Pareek, L. D. Sharma, Jogendra Singh*, R. V. Singh and Vaibhav Sharma \\ Division of Plant Breeding and Genetics, Rajasthan Agricultural Research Institute, \\ S.K.N. Agriculture University, Durgapura (Jaipur), Rajasthan, India-302018 \\ *Corresponding author
}

\begin{abstract}
A B S T R A C T
Keywords

Pearl millet

(Pennisetum

glaucum (L.) R.

Br.), heterosis,

inbreeding

depression, grain

yield, dry fodder

yield

Article Info

Accepted:

05 June 2020

Available Online:

10 July 2020

In the present study, 20 diverse male parents (tester) and 5 female parents (male sterile lines) were used to develop 100 crosses in line $\mathrm{x}$ tester design. The magnitude of heterosis over mid parent value $(\mathrm{H})$, better parent $(\mathrm{HB})$ and hybrid check $(\mathrm{SH})$ for grain yield per plant ranged from $-40.92 \%$ to $37.22 \%,-46.92 \%$ to $34.05 \%$ and $-29 \%$ to $49.13 \%$, respectively in data pooled over three environments, while it were ranged from $-54.37 \%$ to $57.29 \%,-58.77 \%$ to $48.60 \%$ and $-41.97 \%$ to $64.53 \%$, respectively for dry fodder yield per plant. Out of hundred crosses, ten and eleven crosses in pooled data showed significant positive heterosis over hybrid check $(\mathrm{SH})$ for grain yield per plant and dry fodder yield per plant, respectively. Out of these crosses, ICMA-843-22 $x$ RIB-3135-18 (22.37) and ICMA-94111 x RIB- 3135-18 (19.99) found best cross on per se performance for grain yield and also showed significant positive heterosis over standard check (RHB-177) on pooled basis for grain yield (49.13 and 33.27), dry fodder yield per plant (46.42 and 23.39) and earliness of 50\% flowering (-10.29 and 9.29), respectively, thus those crosses could be tested in multi-location programme to identify commercial hybrid.
\end{abstract}

\section{Introduction}

Pearl millet [Pennisetum glaucum (L.) R. Br.] is a nutritious, highly cross-pollinated $\mathrm{C}_{4}$ monocot species belonging to the family Poaceae with protogynous condition and resilient crop in the face of climate change (Anuradha et al., 2017; Sangwan et al., 2019). It is mainly cultivated as a staple food crop in all around the world especially in dry and semi-dry regions of Asia, Africa and Latin
America with more than 30 million hectares (Yadav and Rai, 2013). India is the largest producer of pearl millet, with about 9.1 million metric tons of grains produced from an area of 7.4 million ha (AICPMIP, 2019) with an average productivity of $1,000 \mathrm{~kg} \mathrm{ha}^{-1}$ (Yadav and Rai, 2013).

It is a crop of area characterized with low and erratic rainfall (200-600 mm), high temperature, high salinity or low $\mathrm{pH}$ and 
impoverished infertile soils. Because of its tolerance to harsh growing conditions, it can be grown in areas where other cereal crops, such as wheat, maize and rice would not survive (Sangwan et al., 2019). Besides, it has also a good stover value (used as animal fodder) mainly in north western India, especially in Rajasthan (Yadav and Rai, 2013).

Pearl Millet is grown most widely in India including highly stress prone areas of western Rajasthan. Among the major growing countries India is although having the largest acreage and production but its productivity is the lowest. In the last decades, efforts have been made in India to increase the productivity of pearl millet. This has been possible primarily through the development of hybrid varieties which have yield potential as high as $4000 \mathrm{~kg}$ ha. In spite of the proven superiority of hybrids, their impact on production in the country is by no means dramatic, this mainly because they have not been widely adopted in the principal pearl millet growing areas such as Rajasthan, where hybrid coverage is less. Tendency of farmers to use their own seed saved from the last season's crop, vast fluctuations in yield due to ecological hazards discouraging the farmers to buy costlier hybrid seed, failure of crop establishment particularly in the rainfed arid and semi-arid zones, where the crop is mainly grown, facing the difficulties in seed availability, of some of the factors coming in the way of large scale adoption of hybrids.

Pearl millet is a heterozygous and heterogeneous in nature. Due to its high degree (more than $85 \%$ ) of natural out crossing nature (Burton, 1974), it allows to develop both open-pollinated varieties (OPVs) and single cross hybrids by using partial and fully exploitation of heterosis (Govindaraj et al., 2019). The success of heterosis breeding program in pearl millet is mainly dependent on the development of sustainable cytoplasmic genetic male sterility (CGMS) to develop diverse desirable parents (A/B and R-lines) with wider adaption (including adverse conditions) and greater diversity among them (Satyavathi et al., 2013). Thus, wide genetic variability would be useful to attempt crossing program for these nutritional traits and detect heterotic individuals.

Continued improvement in the yield potential of varieties and hybrids of crop plants depends on selection of parents in hybridization programme. Advancement and diversification of hybrids in pearl millet is a continuous process (Pethani and Kapoor, 1985). The scope for exploitation of hybrid vigour depends on the direction and magnitude of heterosis in the crosses of newly developed parents.

\section{Materials and Methods}

The experimental material for the present study i.e. 100 single cross hybrids $\left(\mathrm{F}_{1}\right.$ `s) generated by crossing 20 genetically diverse inbred lines (male parents) with 5 male sterile lines (female parents) in Line x Tester mating design during Summer, 2018 at RARI, Durgapura and further, the parents along with their $100 \mathrm{~F}_{1}$ 's and two hybrid checks namely, HHB-67 and RHB-177 were evaluated in randomized block design with three replications with three different dates of sowing viz., early sowing ( $\mathrm{E}_{1}-15^{\text {th }}$ June), normal sowing ( $\left.\mathrm{E}_{2}-1^{\text {st }} \mathrm{July}\right)$ and late sowing $\left(\mathrm{E}_{3}-16^{\text {th }}\right.$ July) at RARI, Durgapura, Jaipur, Rajasthan, India during Kharif, 2018. In each environment, plot of parents and $F_{1}$ 's consisted of two rows of 5 meter length with the spacing of $50 \mathrm{~cm}$ between rows and $15 \mathrm{~cm}$ between plants. Normal and uniform cultural operations were followed during the crop season to raise a good crop. To offset the competition effect of hybrids, the inbreds and 
hybrids were separately blocked within each replication (Arunachalam, 1974). Nonexperimental rows planted all around the experiment to safe guard stay animals and birds away. Recommended agronomic practices work followed for raising the crop. Ten competitive plants in parents and $\mathrm{F}_{1}$ 's were selected randomly were tagged before flowering in each plot of every replication for recording observations for all the characters viz., plant height, number of effective tillers per plant, ear length and girth, flag leaf area, dry fodder yield per plant, grain yield per plant, Grain density (no. of grains/ cm squares), 1000-grain weight and harvest index and averaged to obtain the plot mean except, days to $50 \%$ flowering and days to maturity, which were data recorded on plot basis. The mean value of each plot was used for statistical analysis. Analysis of variance for all the characters was done as described by Panse and Sukhatme, (1985) separately for individual as well as for pooled over environments to determine the significance of differences between genotypes, environments and genotype $\mathrm{x}$ environment interaction effects. The percentages of heterosis in $F_{1}$ over the mid-parent (MP) and better parent (BP) were calculated using standard formulae.

For each cross combination, relative midparent heterosis $(\mathrm{H})$ and better-parent heterosis (HB) were calculated as $\mathrm{H}=$ $100 \times\left(\mathrm{F}_{1}-\mathrm{MP}\right) / \mathrm{MP}$ and $\mathrm{HB}=100 \times\left(\mathrm{F}_{1}\right.$ $-\mathrm{BP} / \mathrm{BP}$ respectively. The significance of the heterosis value was identified by the t-test using the error variance of the experiment for all the characters in individual as well as pooled over environments. The significant superiority of hybrid mean was tested by comparing it with the mean performance of the check hybrid HHB-67 and RHB-177, referred as standard heterosis $(\mathrm{SH})$. The mean performance of hybrids one $\mathrm{CD}_{0.05}$ above the best control hybrid was used as a criterion to test their significance.

\section{Results and Discussion}

The pooled analysis of variance over different sowing dates revealed highly significant differences among parents and $\mathrm{F}_{1}$ 's for all the characters studied, indicating the material may therefore, be considered as diverse enough for the study planned. Significant differences among sowing dates were also observed, indicating the differential influence of environment on the character expression. The genotype $\mathrm{x}$ environment interaction was found significant for all characters, indicating existence of non-linear response of genotype to the varying environments.

The possibility of commercial exploitation of hybrid vigor depends on the magnitude of heterosis and feasibility of hybrid seed production. Pearl millet is a cross-pollinated crop and a mechanism of cytoplasmic genetic male sterility is available to produce hybrid seed on a commercial scale. Therefore, the heterosis per se is of economic importance in this crop.

In some cases, crosses with high mid-parent and better-parent heterosis may be found to be inferior to the existing commercial check hybrids. This is attributed to the poor yielding ability of the parents involved in the crosses. In such cases, mid-parent and better-parent heterosis do not hold any economic promise. The check hybrid RHB 177 was considered as the best check to estimate standard heterosis for grain yield as well as for other characters. Therefore, it leads to suggest that the heterosis over the check hybrid (standard heterosis or economic heterosis) should be considered as the most reliable guide for the commercial exploitation of heterosis.

The magnitude of heterosis over mid parent value $(\mathrm{H})$, better parent $(\mathrm{HB})$ and hybrid check (SH) for grain yield per plant ranged from $-40.92 \%$ (ICMA-04999 x RIB-15185) to 
$37.22 \%$ (ICMA-94111 x RIB-3135-18), $46.92 \%$ (ICMA-04999 $\times$ R RIB-15185) to $34.05 \%$ (ICMA-94111 x RIB-3135-18) and $29 \%$ (ICMA-04999 x RIB-15185) to $49.13 \%$ (ICMA-843-22 x RIB-3135-18), respectively on pooled basis (Table 2). Out of hundred crosses, seventeen, nine and ten crosses in pooled data showed significant positive heterosis over mid parent value $(\mathrm{H})$, better parent (HB) and hybrid check (SH), respectively for grain yield per plant. Out of ten crosses, ICMA-843-22 x RIB-3135-18, ICMA-93333 x RIB-15270, ICMA-93333 x RIB-15259 were best crosses showed significant positive heterosis over standard check (RHB-177) on pooled basis for grain yield per plant.

The magnitude of heterosis over mid parent value $(\mathrm{H})$, better parent $(\mathrm{HB})$ and hybrid check $(\mathrm{SH})$ for dry fodder yield per plant ranged from $-54.37 \%$ (ICMA-93333 x RIB15159) to $57.29 \%$ (ICMA-94111 x RIB-494), $-58.77 \%$ (ICMA-93333 x RIB-15159) to $48.60 \%$ (ICMA-94111 x RIB-494) and $41.97 \%$ (ICMA-04999 $\times$ RIB-15185) to 64.53\% (ICMA-843-22 x RIB-192), respectively in data pooled over three environments (Table $2 \& 3$ ). Out of hundred crosses, thirteen, seven, eleven crosses in pooled data showed significant positive heterosis over mid parent value $(\mathrm{H})$, better parent value $(\mathrm{HB})$ and standard check $(\mathrm{SH})$, respectively. Out of eleven, ICMA-843-22 x RIB-192, ICMA-843-22 x RIB-3135-18 and ICMA-94111 x RIB-494 were best among the eleven crosses showed significant positive heterosis over standard check (RHB-177) on pooled basis (Table 3 ).

The results of the present investigation revealed that the relative heterotic expression was fairly high for flag leaf area (20.18 to 108.64), grain density (11.03 to 83.90$)$, plant height (17.20 to 75.10), 1000-grain weight (13.91 to 74.17$)$, harvest index (-29.36 to
66.08), ear girth (17.52 to 65.51), dry fodder yield (-54.37 to 57.29), number of effective tillers per plant (-66.98 to 55.64), ear length (21.53 to 45.09$)$, grain yield per plant $(-40.92$ to 37.22$)$, days to $50 \%$ flowering (-18.86 to $4.50)$ and days to maturity (-10.39 to 1.43$)$ on pooled basis (Table 2).

Maximum desirable heterobeltiosis was 95.59\% for flag leaf area, $82.79 \%$ for grain density, $70.37 \%$ for plant height, $68.24 \%$ for 1000 -grain weight, $63.20 \%$ for harvest index, $53.29 \%$ for ear girth, $48.60 \%$ for dry fodder yield per plant, $44.57 \%$ for ear length, $42.67 \%$ for number of effective tillers per plant, $34.05 \%$ for grain yield per plant, $-18.86 \%$ for days to $50 \%$ flowering and $-10.52 \%$ for days to maturity on pooled basis.

The results are in conformity with the observations of several workers. High estimates of relative heterosis and heterobeltiosis have been reported earlier for plant height (Vetriventhan et al., 2008; Nandaniya et al., 2016); days to $50 \%$ flowering (Vetriventhan et al., 2008; Patel $e t$ al., 2016; Badhe et al., 2018); ear length (Vagadiya et al., 2010; Jethva et al., 2012); ear girth (Nandaniya et al., 2016); flag leaf area (Jethva et al., 2012); dry fodder yield (Jethva et al., 2012; Patel et al., 2016; Badhe et al., 2018); grain yield (Jethva et al., 2012; Nandaniya et al., 2016; Patel et al., 2016; Badhe et al., 2018); 1000-grain weight (Jethva et al., 2012); and harvest index (Jethva et al., 2012; Nandaniya et al., 2016).

Moderate relative heterosis and heterobeltiosis were observed for plant height (Vetriventhan et al., 2008); ear length (Vetriventhan et al., 2008; Badhe et al., 2018); number of effective tillers per plant (Badhe et al., 2018); 1000-grain weight (Nandaniya et al., 2016) and grain yield (Dhuppe et al., 2005; Vetriventhan et al., 2008; Jethva et al., 2012). 
Table.1 ANOVA for grain yield and its components based on data pooled over environments

\begin{tabular}{|c|c|c|c|c|c|c|c|c|c|c|c|c|c|}
\hline \multirow{2}{*}{$\begin{array}{l}\text { Source of } \\
\text { variation }\end{array}$} & \multicolumn{13}{|c|}{ Mean squares } \\
\hline & d.f. & $\begin{array}{c}\text { Days } \\
\text { to } \\
50 \% \\
\text { flowering }\end{array}$ & $\begin{array}{l}\text { Days to } \\
\text { maturity }\end{array}$ & Plant height & $\begin{array}{l}\text { No. of } \\
\text { effective } \\
\text { tillers / } \\
\text { plant }\end{array}$ & Ear length & $\begin{array}{l}\text { Ear- } \\
\text { Girth }\end{array}$ & $\begin{array}{c}\text { Flag leaf } \\
\text { Area }\end{array}$ & $\begin{array}{c}\text { Dry Fodder } \\
\text { yield / } \\
\text { plant }\end{array}$ & $\begin{array}{l}\text { Grain yield } \\
\text { / plant }\end{array}$ & $\begin{array}{c}\text { Grain } \\
\text { Density }\end{array}$ & $\begin{array}{l}1000- \\
\text { grain } \\
\text { weigh }\end{array}$ & $\begin{array}{c}\text { Harvest } \\
\text { index }\end{array}$ \\
\hline Env. & 2 & $6119.51 * *$ & $5230.84 * *$ & $160448.75^{* *}$ & $5.3^{* *}$ & $985.77 * *$ & $35.55 * *$ & $92755.19 * *$ & $84640.83 * *$ & $11513.72 * *$ & $3646.07 * *$ & $1159.53 * *$ & $12528.52 * *$ \\
\hline Replication(r) & 6 & 3.75 & 3.45 & 94.33 & $0.20 *$ & 3.44 & 0.03 & 35.41 & 27.75 & 14.18 & 14.21 & 0.75 & 14.99 \\
\hline Genotype (G) & 124 & $39.20 * *$ & $34.72 * *$ & $5262.99 * *$ & $2.64 * *$ & $52.22 * *$ & $0.60 * *$ & $2268.61 * *$ & $665.27 * *$ & $45.31 * *$ & $232.46 * *$ & $15.88 * *$ & $204.12 * *$ \\
\hline Parents (P) & 24 & $67.48 * *$ & $71.08 * *$ & $1155.00 * *$ & $5.89 * *$ & $118.28 * *$ & $0.08 * *$ & $646.06 * *$ & $1156.84 * *$ & $31.54 * *$ & $144.15^{* *}$ & $2.45^{*}$ & $252.44 * *$ \\
\hline Hybrids (H) & 99 & $13.58 * *$ & $11.17 * *$ & $426.49 * *$ & $0.62 * *$ & $20.82 * *$ & $0.09 * *$ & $1145.87 * *$ & $506.72 * *$ & $48.91 * *$ & $76.98 * *$ & $5.30 * *$ & $179.22 * *$ \\
\hline P vs H & 1 & $1947.49 * *$ & $1540.33 * *$ & $592341.85^{* *}$ & $128.95 * *$ & $1638.48 * *$ & $64.71 * *$ & $154606.56 * *$ & $4881.40 * *$ & $12.30 * *$ & $18056.35 * *$ & $1407.11 * *$ & $1559.35^{* *}$ \\
\hline $\mathbf{G} \times \mathbf{E}$ & 252 & $13.26 * *$ & $10.88 * *$ & $278.73 * *$ & $0.42 * *$ & $7.37 * *$ & $0.05^{* *}$ & $677.66^{* *}$ & $232.40 * *$ & $26.39 * *$ & $38.42 * *$ & $3.31 * *$ & $85.03 * *$ \\
\hline Error & 756 & 3.99 & 3.57 & 179.80 & 0.09 & 3.47 & 0.02 & 233.38 & 25.86 & 6.05 & 15.60 & 1.47 & 18.71 \\
\hline
\end{tabular}

$*, * *$ Significant at $5 \%$ and $1 \%$ level of significance, respectively

Table.2 Range of heterosis and number of desirable crosses for grain yield and its contributing characters based on pooled over environments

\begin{tabular}{|c|c|c|c|c|c|c|}
\hline \multirow[t]{2}{*}{ Characters } & \multicolumn{3}{|c|}{ Range of heterosis (\%) } & \multicolumn{3}{|c|}{$\begin{array}{c}\text { Number of crosses showing desirable } \\
\text { significant heterosis }\end{array}$} \\
\hline & $\mathbf{H}$ & HB & SH & $\mathbf{H}$ & HB & SH \\
\hline Days to $50 \%$ flowering & $(-) 18.86-4.50$ & $(-) 18.86-4.33$ & $(-) 10.29-(-) 7.28$ & 70 & 76 & 5 \\
\hline Days to maturity & $(-) 10.39-1.43$ & $(-) 10.52-2.41$ & $(-) 4.49-3.15$ & 67 & 74 & 1 \\
\hline Plant height & $17.20-75.10$ & $7.77-70.37$ & $14.00-22.23$ & 100 & 87 & 29 \\
\hline No. of effective tillers / plant & $(-) 66.98-55.64$ & $(-) 71.81-42.67$ & $(-) 38.27-(-) 32.72$ & 3 & 2 & 0 \\
\hline Ear length & $(-) 21.53-45.09$ & $(-) 35.05-44.57$ & $(-) 30.91-17.01$ & 51 & 7 & 1 \\
\hline Ear girth & $17.52-65.51$ & $2.04-53.29$ & $10.19-20.95$ & 100 & 100 & 27 \\
\hline Flag leaf Area & $20.18-108.64$ & $18.17-95.59$ & $(-) 29.99-(-) 23.53$ & 87 & 76 & 0 \\
\hline Dry Fodder yield / plant & $(-) 54.37-57.29$ & $(-) 58.77-48.60$ & $(-) 41.97-64.53$ & 13 & 6 & 12 \\
\hline Grain yield / plant & $(-) 40.92-37.22$ & $(-) 46.92-34.05$ & $(-) 29.00-49.13$ & 17 & 9 & 10 \\
\hline Grain Density & $11.03-83.90$ & $(-) 27.52-82.71$ & $(-) 26.18-(-) 20.89$ & 82 & 60 & 0 \\
\hline 1000-grain weight & $13.91-72.17$ & $14.34-68.24$ & $24.28-43.09$ & 97 & 92 & 38 \\
\hline Harvest index & $(-) 29.36-66.08$ & $(-) 44.11-63.20$ & $(-) 34.31-37.56$ & 53 & 32 & 15 \\
\hline
\end{tabular}


Table.3 Estimates of heterosis $(\mathrm{H})$, heterobeltiosis $(\mathrm{HB})$, and standard heterosis $(\mathrm{SH})$ for dry fodder yield per plant and grain yield per plant data pooled over three environments

\begin{tabular}{|c|c|c|c|c|c|c|c|c|c|}
\hline \multirow[t]{2}{*}{ SN } & \multirow[t]{2}{*}{ Crosses } & \multicolumn{4}{|c|}{ Grain yield /plant (g) } & \multicolumn{4}{|c|}{ Dry fodder yield/plant (g) } \\
\hline & & Mean & H & HB & SH & Mean & H & HB & SH \\
\hline 1 & ICMA 93333 X RIB-192 & 15.6 & $-11.92 *$ & $-22.76^{* *}$ & 4.00 & 33.43 & -5.74 & $-30.45 * *$ & -7.14 \\
\hline 2 & ICMA 93333 X RIB-494 & 15.46 & -7.28 & $-23.43 * *$ & 3.07 & 28.02 & $-28.01 * *$ & $-41.71 * *$ & -22.17 \\
\hline 3 & ICMA 93333 X RIB-3135-18 & 16.85 & -4.01 & $-16.55 * *$ & 12.33 & 32.78 & $-19.02 * *$ & $31.80 * *$ & -8.94 \\
\hline 4 & ICMA 93333 X RIB-135071 & 12.48 & $-26.93 * *$ & $-38.17 * *$ & -16.8 & 25.88 & $-36.90 * *$ & $-46.15 * *$ & $-28.11^{*}$ \\
\hline 5 & ICMA 93333 X MIR-525-2 & 18.53 & 4.39 & -8.22 & 23.53 & 36.47 & $-11.79 *$ & $-24.13 * *$ & 1.31 \\
\hline 6 & ICMA 93333 X RIB-155076 & 16.05 & -7.29 & $-20.48 * *$ & 7.00 & 27.52 & $-34.95 * *$ & $-42.74 * *$ & $-23.56^{*}$ \\
\hline 7 & ICMA 93333 X RIB-155137 & 16.74 & -5.67 & $-17.06 * *$ & 11.60 & 29.32 & $-35.82 * *$ & $-38.99 * *$ & -18.56 \\
\hline 8 & ICMA 93333 X RIB-155147 & 15.69 & -9.08 & $-22.26 * *$ & 4.60 & 29.98 & $-23.59 * *$ & $-37.63 * *$ & -16.72 \\
\hline 9 & ICMA 93333 X J-2290 & 13.88 & $-24.42 * *$ & $-30.23 * *$ & -6.07 & 27.67 & $-27.65 * *$ & $-33.70 * *$ & -11.47 \\
\hline 10 & ICMA 93333 X J-2340 & 15.32 & 0.30 & -5.30 & $27.47 *$ & 24.56 & $-13.40^{*}$ & $-28.57 * *$ & -4.64 \\
\hline 11 & ICMA 93333 X H-77/833-2 & 17.75 & $-11.50^{*}$ & $-16.57 * *$ & 12.33 & 32.51 & $-38.27 * *$ & $-47.11 * *$ & $-29.36^{*}$ \\
\hline 12 & ICMA 93333 X RIB-15153 & 12.96 & $-19.48 * *$ & $-31.24 * *$ & -7.47 & 23.25 & $-41.62 * *$ & $-42.43 * *$ & $-23.14^{*}$ \\
\hline 13 & ICMA 93333 X RIB-15159 & 17.02 & $-14.70 * *$ & $-24.12 * *$ & 2.13 & 34.7 & $-54.37 * *$ & $-58.77 * *$ & $-31.78^{* *}$ \\
\hline 14 & ICMA 93333 X RIB-15181 & 15.43 & -2.34 & $-12.05^{*}$ & 18.33 & 30.47 & $-44.33 * *$ & $-52.69 * *$ & -9.69 \\
\hline 15 & ICMA 93333 X RIB-15185 & 13.17 & $-35.19 * *$ & $-35.78 * *$ & -13.60 & 37.11 & $-44.85^{* *}$ & $-51.63 * *$ & $-35.42 * *$ \\
\hline 16 & ICMA 93333 X RIB-15197 & 14.09 & $-11.99^{*}$ & $-15.68 * *$ & 13.47 & 31.87 & $-23.56^{* *}$ & $-27.81 * *$ & -3.61 \\
\hline 17 & ICMA 93333 X RIB-15217 & 19.12 & $-15.54 * *$ & $-23.58 * *$ & 2.87 & 34.33 & $-25.12 * *$ & $-36.62 * *$ & -15.36 \\
\hline 18 & ICMA 93333 X RIB-15243 & 16.85 & $-23.32 * *$ & $-34.76 * *$ & -12.20 & 25.43 & -3.47 & -22.79 & 3.08 \\
\hline 19 & ICMA 93333 X RIB-15259 & 19.27 & 7.97 & -4.54 & $28.47 *$ & 32.33 & $-19.79 * *$ & $-32.73 * *$ & -10.19 \\
\hline 20 & ICMA 93333 X RIB-15270 & 20.77 & $11.85^{*}$ & 2.89 & $38.47 * *$ & 37.68 & -9.05 & $-21.62 * *$ & 4.67 \\
\hline 21 & ICMA-843-22 x RIB-192 & 19.05 & $12.82 *$ & 2.71 & $27.00 *$ & 59.23 & $32.31 * *$ & $-11.16^{* *}$ & $64.53 * *$ \\
\hline 22 & ICMA-843-22 x RIB-494 & 16.9 & 6.63 & -8.86 & 12.67 & 39.29 & $-18.53 * *$ & $-41.07 * *$ & 9.14 \\
\hline 23 & ICMA-843-22 x RIB-3135-18 & 22.37 & $33.72 * *$ & $20.62 * *$ & $49.13 * *$ & 52.71 & 5.89 & $-20.93 * *$ & $46.42 * *$ \\
\hline 24 & ICMA-843-22 x RIB-135071 & 14.99 & -7.81 & $-19.16^{* *}$ & -0.07 & 38.57 & $-23.34 * *$ & $-42.14 * *$ & 7.14 \\
\hline 25 & ICMA-843-22 x MIR-525-2 & 18.28 & 7.97 & -1.44 & 21.87 & 48.18 & -4.87 & $-27.73 * *$ & $33.83 * *$ \\
\hline 26 & ICMA-843-22 x RIB-155076 & 14.57 & -11.69 & $-21.45 * *$ & -2.87 & 39.05 & $-24.34 * *$ & $-41.43 * *$ & 8.47 \\
\hline 27 & ICMA-843-22 x RIB-155137 & 14.12 & -16.60 & $-23.87 * *$ & -5.87 & 29.9 & $-45.63 * *$ & $-55.16 * *$ & -16.94 \\
\hline 28 & ICMA-843-22 x RIB-155147 & 13.85 & $-15.78^{*}$ & $-25.34 * *$ & -7.67 & 28.21 & $-41.87 * *$ & $-57.68 * *$ & -21.64 \\
\hline 29 & ICMA-843-22 x J-2290 & 12.76 & $-19.39 * *$ & $-22.55 * *$ & -4.27 & 30.38 & $-38.76^{* *}$ & $-51.00 * *$ & -9.25 \\
\hline 30 & ICMA-843-22 x J-2340 & 15.12 & $-11.59 *$ & $-1305^{*}$ & 7.47 & 32.95 & -6.56 & $-31.40 * *$ & $27.03 *$ \\
\hline 31 & ICMA-843-22 x H-77/833-2 & 14.38 & $-14.04 *$ & $-15.58 *$ & 4.33 & 33.56 & $-17.72 * *$ & $-37.69 * *$ & 15.39 \\
\hline 32 & ICMA-843-22 x RIB-15153 & 11.82 & -22.27 & $-31.19 * *$ & -14.93 & 42.92 & $-46.43 * *$ & $-54.43 * *$ & -15.61 \\
\hline 33 & ICMA-843-22 x RIB-15159 & 14.54 & $-11.8^{*}$ & $-18.48 * *$ & 0.80 & 41.84 & $-47.79 * *$ & $-50.58 * *$ & -8.47 \\
\hline 34 & ICMA-843-22 x RIB-15181 & 12.62 & $17.13^{* *}$ & $-22.43 * *$ & -4.13 & 45.8 & $-50.42 * *$ & $-51.16 * *$ & -6.78 \\
\hline 35 & ICMA-843-22 x RIB-15185 & 14.91 & $-38.37 * *$ & $-40.35 * *$ & -21.2 & 31.91 & $-16.60 * *$ & $-35.62 * *$ & 19.22 \\
\hline 36 & ICMA-843-22 x RIB-15197 & 14.36 & $-21.51 * *$ & $-21.62 * *$ & -3.07 & 32.67 & $-23.50 * *$ & $-37.23 * *$ & 16.22 \\
\hline 37 & ICMA-843-22 x RIB-15217 & 16.12 & $-27.64 * *$ & $-31.94 * *$ & -15.87 & 45.73 & $-8.39 *$ & $-31.30 * *$ & $27.22 *$ \\
\hline 38 & ICMA-843-22 x RIB-15243 & 15.65 & -8.85 & $-19.60 * *$ & -0.6 & 41.54 & $-33.16^{* *}$ & $-52.13 * *$ & -11.36 \\
\hline 39 & ICMA-843-22 x RIB-15259 & 15.53 & -8.80 & $-16.26^{* *}$ & 3.53 & 34.08 & $-31.30 * *$ & $-48.88 * *$ & -5.33 \\
\hline 40 & ICMA-843-22 x RIB-15270 & 17.73 & -0.14 & -4.42 & 18.20 & 45.25 & $-10.80 * *$ & $-32.13 * *$ & $25.69 *$ \\
\hline 41 & ICMA-97111 x RIB-192 & 14.74 & -0.38 & -9.41 & -1.73 & 42.31 & $38.47 * *$ & 10.62 & 17.53 \\
\hline 42 & ICMA-97111 x RIB-494 & 16.13 & 9.63 & -0.89 & 7.53 & 36.38 & 6.96 & -4.89 & 1.06 \\
\hline 43 & ICMA-97111 x RIB-3135-18 & 18.65 & $19.64 * *$ & $14.62 *$ & 24.33 & 39.78 & $11.82 *$ & 3.99 & 10.5 \\
\hline
\end{tabular}




\begin{tabular}{|c|c|c|c|c|c|c|c|c|c|}
\hline 44 & ICMA-97111 x RIB-135071 & 17.42 & $15.17 *$ & 7.04 & 16.13 & 25.92 & $-28.22 * *$ & $-32.24 * *$ & $-28.00 *$ \\
\hline 45 & ICMA-97111 x MIR-525-2 & 13.75 & $-12.94 *$ & $-15.53 *$ & -8.33 & 30.87 & $-15.28 * *$ & $-19.30^{* * *}$ & -14.25 \\
\hline 46 & ICMA-97111 x RIB-155076 & 14.36 & -6.50 & -11.77 & -4.27 & 32.78 & $-12.37^{*}$ & $-14.31 *$ & -8.94 \\
\hline 47 & ICMA-97111 x RIB-155137 & 15.24 & -3.48 & -6.35 & 1.60 & 24.38 & $-40.21 * *$ & $-43.70 * *$ & $-32.28 * *$ \\
\hline 48 & ICMA-97111 x RIB-155147 & 15.39 & 0.56 & -5.45 & 2.60 & 33.93 & -1.16 & -11.31 & -5.75 \\
\hline 49 & ICMA-97111 x J-2290 & 13 & $-22.05 * *$ & $-23.90 * *$ & -13.33 & 35.11 & -10.32 & $-12.31 *$ & -2.47 \\
\hline 50 & ICMA-97111 x J-2340 & 15.67 & -8.4 & -12.63 & 4.47 & 39.84 & $14.69 *$ & 4.14 & 10.67 \\
\hline 51 & ICMA-97111 x H-77/833-2 & 15.73 & -7.87 & -12.00 & 4.87 & 45.91 & $26.55^{* *}$ & $20.00^{* *}$ & $27.53 *$ \\
\hline 52 & ICMA-97111 x RIB-15153 & 16.37 & 7.12 & 0.57 & 9.13 & 32.04 & $-24.62 * *$ & $-31.47 * *$ & -11.00 \\
\hline 53 & ICMA-97111 x RIB-15159 & 13.68 & $-14.54 *$ & $-15.97 *$ & -8.80 & 28.96 & $-40.79 * *$ & $-51.38 * *$ & -19.56 \\
\hline 54 & ICMA-97111 x RIB-15181 & 18.08 & 11.41 & 11.05 & 20.53 & 32.46 & $-39.32 * *$ & $-52.77 * *$ & -9.83 \\
\hline 55 & ICMA-97111 x RIB-15185 & 15.12 & $-16.25 * *$ & $-23.74 * *$ & 0.80 & 21.21 & $-43.08 * *$ & $-44.57 * *$ & $-41.08 * *$ \\
\hline 56 & ICMA-97111 x RIB-15197 & 14.03 & $-19.32 * *$ & $-24.16 * *$ & -6.47 & 38.29 & -5.44 & -10.38 & 6.36 \\
\hline 57 & ICMA-97111 x RIB-15217 & 16.79 & 2.94 & 2.73 & 11.93 & 40.15 & $12.18^{*}$ & 4.93 & 11.53 \\
\hline 58 & ICMA-97111 x RIB-15243 & 14.95 & -1.82 & -8.18 & -0.33 & 44.5 & $32.70^{* *}$ & $16.34 * *$ & $23.61 *$ \\
\hline 59 & ICMA-97111 x RIB-15259 & 14.29 & -10.09 & -12.21 & -4.73 & 37.87 & 6.97 & -1.00 & 5.19 \\
\hline 60 & ICMA-97111 x RIB-15270 & 16.97 & 2.15 & 0.10 & 13.13 & 40 & 9.50 & 4.57 & 11.11 \\
\hline 61 & ICMA-04999 x RIB-192 & 17.41 & 10.71 & 7.30 & 16.07 & 36.19 & 9.54 & $-16.24 * *$ & 0.53 \\
\hline 62 & ICMA-04999 x RIB-494 & 14.23 & -3.15 & -12.31 & -5.13 & 30.45 & $-16.56^{* *}$ & $-29.52 * *$ & -15.42 \\
\hline 63 & ICMA-04999 x RIB-3135-18 & 15.53 & -0.21 & -4.24 & 3.53 & 34.06 & -10.49 & $-21.17 * *$ & -5.39 \\
\hline 64 & ICMA-04999 x RIB-135071 & 11.6 & $-23.22 * *$ & $-28.53 * *$ & -22.67 & 30.95 & $-19.78 * *$ & $-28.36 * *$ & -14.03 \\
\hline 65 & ICMA-04999 x MIR-525-2 & 15.87 & 0.66 & -2.16 & 5.80 & 26.5 & $-31.88 * *$ & $-38.65 * *$ & $-26.39 *$ \\
\hline 66 & ICMA-04999 x RIB-155076 & 16.51 & 7.67 & 1.77 & 10.07 & 28.26 & $-29.13 * *$ & $-34.58 * *$ & -21.5 \\
\hline 67 & ICMA-04999 x RIB-155137 & 14.37 & -8.88 & -11.44 & -4.20 & 24.83 & $-42.58 * *$ & $-42.66 * *$ & $-31.03 * *$ \\
\hline 68 & ICMA-04999 x RIB-155147 & 12.55 & $-17.84 * *$ & $-22.62 * *$ & -16.33 & 20.89 & $-43.23 * *$ & $-51.64 * *$ & $-41.97 * *$ \\
\hline 69 & ICMA-04999 x J-2290 & 15.67 & -5.90 & -8.28 & 4.47 & 23.45 & $-43.66^{* *}$ & $-45.73 * *$ & $-34.86 * *$ \\
\hline 70 & ICMA-04999 x J-2340 & 17.05 & -0.18 & -4.94 & 13.67 & 24.99 & $-32.85 * *$ & $-42.16 * *$ & $-30.58 * *$ \\
\hline 71 & ICMA-04999 x H-77/833-2 & 12.21 & $-28.37 * *$ & $-31.68 * *$ & -18.60 & 25.71 & $-33.66 * *$ & $-40.49 * *$ & $-28.58^{*}$ \\
\hline 72 & ICMA-04999 x RIB-15153 & 15.56 & 1.96 & -4.12 & 3.73 & 32.82 & $-27.03 * *$ & $-29.80 * *$ & -8.83 \\
\hline 73 & ICMA-04999 x RIB-15159 & 11.46 & $-28.28 * *$ & $-29.36 * *$ & -23.60 & 25 & $-51.35^{* *}$ & $-58.03 * *$ & $-30.56 * *$ \\
\hline 74 & ICMA-04999 x RIB-15181 & 11.65 & $-28.10 * *$ & $-28.21 * *$ & -22.33 & 29.2 & $-47.81 * *$ & $-57.50 * *$ & -18.89 \\
\hline 75 & ICMA-04999 x RIB-15185 & 10.65 & $-40.92 * *$ & $-46.29 * *$ & $-29.00 *$ & 24.44 & $-38.47 * *$ & $-43.42 * *$ & $-32.11 * *$ \\
\hline 76 & ICMA-04999 x RIB-15197 & 14.06 & $-19.00 * *$ & $-23.97 * *$ & -6.27 & 31.4 & $-26.92 * *$ & $-27.32 * *$ & -12.78 \\
\hline 77 & ICMA-04999 x RIB-15217 & 14.14 & $-13.15^{*}$ & -13.47 & -5.73 & 25.59 & $-33.12 * *$ & $-40.77 * *$ & $-28.92 *$ \\
\hline 78 & ICMA-04999 x RIB-15243 & 14.93 & -1.72 & -7.95 & -0.47 & 28.78 & $-20.09 * *$ & $-33.38 * *$ & -20.06 \\
\hline 79 & ICMA-04999 x RIB-15259 & 15.1 & -4.82 & -6.90 & 0.67 & 22.1 & $-41.66 * *$ & $-48.85 * *$ & $-38.61 * *$ \\
\hline 80 & ICMA-04999 x RIB-15270 & 14.44 & $-12.99 *$ & $-14.87 *$ & -3.73 & 25.71 & $-34.06 * *$ & $-40.49 * *$ & $-28.58^{*}$ \\
\hline 81 & ICMA-94111 x RIB-192 & 18.33 & $24.51 * *$ & $20.42 * *$ & 22.2 & 42.79 & $51.88 * *$ & $27.81 * *$ & 18.86 \\
\hline 82 & ICMA-94111 x RIB-494 & 16.27 & $18.86^{*}$ & 14.4 & 8.47 & 49.75 & $57.29 * *$ & $48.60 * *$ & $38.19 * *$ \\
\hline 83 & ICMA-94111 x RIB-3135-18 & 19.99 & $37.22 * *$ & $34.05 * *$ & $33.27 *$ & 44.42 & $33.84 * *$ & $32.69 * *$ & $23.39 *$ \\
\hline 84 & ICMA-94111 x RIB-135071 & 19.03 & $34.95 * *$ & $33.79 * *$ & $26.87 *$ & 35.76 & 6.05 & 5.29 & -0.67 \\
\hline 85 & ICMA-94111 x MIR-525-2 & 17.12 & $15.93^{*}$ & 11.81 & 14.13 & 39.42 & $15.75^{*}$ & $13.84 *$ & 9.50 \\
\hline 86 & ICMA-94111 x RIB-155076 & 18.5 & $29.06^{* *}$ & $28.07 * *$ & 23.33 & 37.83 & 8.03 & 3.48 & 5.08 \\
\hline 87 & ICMA-94111 x RIB-155137 & 16.47 & 11.56 & 7.59 & 9.80 & 45.5 & $18.51 * *$ & 5.06 & $26.39 *$ \\
\hline 88 & ICMA-94111 x RIB-155147 & 14.19 & -0.59 & -0.98 & -5.40 & 37.82 & $18.40 * *$ & 12.96 & 5.06 \\
\hline 89 & ICMA-94111 x J-2290 & 12.91 & $-17.54 * *$ & $-24.45 * *$ & -13.93 & 28.35 & $-22.86 * *$ & $-29.18 * *$ & -21.25 \\
\hline 90 & ICMA-94111 x J-2340 & 17.97 & 11.78 & 0.22 & 19.80 & 35.03 & 8.29 & 4.64 & -2.69 \\
\hline 91 & ICMA-94111 x H-77/833-2 & 19.58 & $22.01 * *$ & 9.53 & $30.53 *$ & 30.28 & -10.65 & -11.72 & -15.89 \\
\hline
\end{tabular}




\begin{tabular}{|l|l|l|l|l|l|l|l|l|c|c|}
\hline $\mathbf{9 2}$ & ICMA-94111 x RIB-15153 & 15.41 & 8.13 & 7.88 & 2.73 & 32.29 & $-19.50 * *$ & $-32.92 * *$ & -10.31 \\
\hline $\mathbf{9 3}$ & ICMA-94111 x RIB-15159 & 19.64 & $31.14 * *$ & $24.84 * *$ & $30.93 *$ & 44.48 & -4.39 & $-25.32 * *$ & $23.56 *$ \\
\hline $\mathbf{9 4}$ & ICMA-94111 x RIB-15181 & 15.56 & 2.39 & -3.79 & 3.73 & 37.83 & $-25.95 * *$ & $-44.94 * *$ & 5.08 \\
\hline $\mathbf{9 5}$ & ICMA-94111 x RIB-15185 & 17.65 & 3.67 & -10.98 & 17.67 & 28.79 & $-17.43 * *$ & $-20.60 * *$ & -20.03 \\
\hline $\mathbf{9 6}$ & ICMA-94111 x RIB-15197 & 19.22 & $17.49 * *$ & 3.92 & $28.13 *$ & 31.44 & $-17.47 * *$ & $-26.40 * *$ & -12.67 \\
\hline $\mathbf{9 7}$ & ICMA-94111 x RIB-15217 & 18.74 & $22.62 * *$ & $14.66 *$ & 24.93 & 25.4 & $-23.94 * *$ & $-24.13 * *$ & $-29.44 *$ \\
\hline $\mathbf{9 8}$ & ICMA-94111 x RIB-15243 & 16.89 & $19.01 * *$ & $18.78^{*}$ & 12.60 & 31.42 & 0.88 & -6.14 & -12.72 \\
\hline $\mathbf{9 9}$ & ICMA-94111 x RIB-15259 & 15.49 & 4.16 & -0.16 & 3.27 & 31.94 & -3.28 & -4.61 & -11.28 \\
\hline $\mathbf{1 0 0}$ & ICMA-94111 x RIB-15270 & 16.6 & 6.51 & -2.07 & 10.67 & 36.63 & 7.33 & 5.33 & 1.75 \\
\hline & S.E.D & 15.89 & 1.00 & 1.16 & & 35.15 & 2.07 & 2.39 & \\
\hline & CD 5\% & 1.14 & 1.97 & 2.27 & & 5.62 & 4.07 & 4.7 & \\
\hline & CD 1\% & 3.17 & 2.59 & 2.99 & & 15.6 & 5.36 & 6.19 & \\
\hline
\end{tabular}

Among the characters, maximum number of crosses with significant desirable standard heterosis was found in plant height, ear girth, test weight, harvest index, dry fodder yield per plant and grain yield per plant. When crosses were compared individually for each character, highest desirable heterotic expression (over superior check hybrid) was for dry fodder yield per plant (64.53) in cross ICMA- 843-22 x RIB-192 followed by grain yield per plant (49.13) in cross ICMA-843-22 $x$ RIB-3135-18; 1000-grain weight (43.09) in cross ICMA-94111 x RIB-494; harvest index (37.56) in cross ICMA-94111 x RIB-15217; plant height (22.23) in cross ICMA-93333 x RIB- 15243; ear girth (20.95) in cross ICMA93333 x RIB- 15185. Earliness is the desirable character, and therefore, in the present study the cross ICMA-94111 x RIB$3135-18$ is early in maturity and the crosses ICMA- 843-22 x RIB-3135-18, ICMA- 843$22 \times$ RIB-155137 and ICMA- $9711 \times$ RIB3135-18 were considered very desirable as it showed standard heterosis for earliness in flowering.

High estimation of standard heterosis has been reported plant height (Vetriventhan et al., 2008; Patel et al., 2016; Salagarkar and Wali, 2016; Badhe et al., 2018); number of effective tillers and 1000-grain weight (Jethva et al., 2012); harvest index (Kumar et al., 2017); dry fodder yield (Jethva et al., 2012; Badhe et al., 2018); and grain yield (Jethva et al., 2012; Kumar et al., 2017; Badhe et al., 2018; Kumawat et al., 2019). In the pooled analysis, 10 crosses showed significant positive standard heterosis for grain yield per plant (Table 3.). These 10 crosses viz., ICMA-843-22 x RIB-3135-18, ICMA-93333 $x$ RIB-15270, ICMA-94111 x RIB- 3135-18, ICMA-94111 x RIB-15159, ICMA-94111 x H-77/833-2， ICMA-93333 x RIB-15259, ICMA-94111 x RIB-15197, ICMA-93333 x J-2340, ICMA-843-22 x RIB-192 and ICMA$94111 \times$ RIB-135071 have high per se performance. Above crosses were also found to be superior in there per se performance for various contributing traits. Out of these crosses in per se performance for grain yield per plant, cross ICMA-843-22 x RIB-3135-18 and ICMA-94111 x RIB- 3135-18 having high positive standard heterosis for grain yield per plant as well as dry fodder yield per plant and earliness of 50\% flowering while cross ICMA 93333 x RIB-15270 having high positive standard heterosis for grain yield per plant only, thus these crosses could be tested in multi-location to identify commercial hybrid.

\section{References}

AIRCP on Pearl Millet - Report on the Project Coordinator Review. 2019. 54th Annual Group Meeting. ICAR- Indian Agricultural Research Institute, New Delhi.

Anuradha, N., Satyavathi, C.T., Meena, M.C., 
Sankar, S.M., Bharadwaj, C., Bhat, J., Singh, O., Singh, S.P. 2017. Evaluation of pearl millet (Pennisetum glaucum (L.) R. Br.) for grain iron and zinc content in different agro climatic zones of India. Indian J. Genet. Plant Breed., 77: 65-73.

Arunachalam, V. 1974. The fallacy behind the use of a modified line $\mathrm{x}$ tester design. Indian $\mathrm{J}$. Genet., 34: 280-287.

Badhe, P. L., Patil, H. T., Borole, D. N., \& Thakare, S. M. 2018. Heterosis for yield and morpho-nutritional traits in pearl millet [Pennisetum glaucum (1.) R. Br.]. Elect. J. Plant Breed., 9 (2): 759-762.

Burton, G.W. 1974. Factor affecting pollen movement and natural crossing in pearl millet. Crop Sci., 14: 802-805.

Dhuppe, M.V., Chavan, A.A., More, A.W., More, S.S. and Choudhary, L.M. 2005. Heterosis in pearl millet. J. Soils Crops, 15 (2): 343-351.

Govindaraj, M., Rai, K.N., Kanatti, A., Rao, A.S. and Shivade. H. 2019. Nutritional security in drylands: Fast-track intra-population genetic improvement for grain iron and zinc densities in pearl millet. Front. Nutr. 6: 74.

Jethva, A.S., Raval, L., Madariya, R.B., Mehta, D.R. and Mandavia, C. 2012. Heterosis for grain yield and its related characters in pearl millet. Elect. J. Plant Breed., 3 (3): 848-852.

Kumar, M., Gupta, P. C., Sharma, N., and Sharma, A. K. 2017. Estimation of standard heterosis for grain yield and yield components in pearl millet (Pennisetum glaucum (L.) R. Br.). J. Pharmacognosy Phytochem., 6 (4): 785-788.

Kumawat, K.R., Gupta, P.C. and Sharma, N.K. 2019. Identification of pearl millet [Pennisetum glaucum (L.) R. Br.] heterotic crosses involving cytoplasmic-genetic male sterility in low rainfall areas. Chem. Sci. Rev. Lett., 8 (30): 272-275.

Nandaniya K.U., Mungra, K.D. and Sorathiya, J.S. 2016. Estimation of heterosis in pearl millet [Pennisetum glaucum (L.)] for yield and quality traits. Elect. J. Plant Breed., 7(3): 758-760.

Panse, V.G. and Sukhatme, P.V. (1985). Statistical methods for agricultural workers. ICAR, New Delhi, pp. 381.

Patel, B.C., Doshi, J.S. and Patel, J.A. 2016. Heterosis for grain yield components in pearl millet (Pennisetum glaucum (L.) R. Br.). Innovare J. Agri. Sci., 4 (3): 1-3.

Pethani, K.V. and Kapoor, R.L. 1985. Phenotypic stability for grain yield in pearl millet. Indian J. Genet., 45 (2): 362-367.

Salagarkar, S. and Wali, M.C. 2016. Heterosis for yield and yield related components using diverse restorer lines in pearl millet [Pennisetum glaucum (L.) R. Br.]. J. Farm Sci., 29 (4): 436-438.

Sangwan, S., Yashveer, S., Kumar, R., Hamender, Sharma, S. and Redhu, N. 2019. Multi trait analysis reveals substantial diversity in pearl millet [Pennisetum glaucum (L.) R. Br.] inbred lines. J. Exp. Biol. Agric. Sci., 7(4): $358-375$.

Satyavathi, C.T., Tiwari, S., Bharadwaj, C., Rao, A.R., Bhat, J. and Singh, S.P. 2013. Genetic diversity analysis in a novel set of restorer lines of pearl millet (Pennisetum glaucum (L.) R. Br) using SSR markers. Vegetos. 26, 72-82.

Vagadiya, K.J., Dhedhi, K.K., Joshi, H.J., Bhadelia, A.S. and Vekariya, H.B. 2010. Studies on Heterosis in Pearl Millet [Pennisetum glaucum (L.) R. BR.]. Agric. Sci. Digest, 30 (3): 197201.

Vetriventhan, M., Nirmalakumari, A., and Ganapathy, S. 2008. Heterosis for grain yield components in Pearl Millet (Pennisetum glaucum (L.) R. Br.). World J. Agric. Sci., 4 (5): 657-660.

Yadav, O.P. and Rai, K.N. 2013. Genetic improvement of pearl millet in India. Agric. Res., 2: 275-292.

\section{How to cite this article:}

Anita Pareek, L. D. Sharma, Jogendra Singh, R. V. Singh and Vaibhav Sharma. 2020. Heterosis Studies for Grain Yield and its Contributing Traits in Pearl millet (Pennisetum glaucum (L.) R. Br.) under Different Sowing Conditions. Int.J.Curr.Microbiol.App.Sci. 9(07): 520-528. doi: https://doi.org/10.20546/ijcmas.2020.907.057 